Macroscopic effects of tunnelling barriers in aggregates of carbon nanotube bundles

This article has been downloaded from IOPscience. Please scroll down to see the full text article.

2012 J. Phys. D: Appl. Phys. 45105306

(http://iopscience.iop.org/0022-3727/45/10/105306)

View the table of contents for this issue, or go to the journal homepage for more

Download details:

IP Address: 141.108.250.211

The article was downloaded on 27/02/2012 at 08:54

Please note that terms and conditions apply. 


\title{
Macroscopic effects of tunnelling barriers in aggregates of carbon nanotube bundles
}

\author{
M Salvato ${ }^{1}$, M Cirillo ${ }^{1}$, M Lucci $^{1}$, S Orlanducci $^{2}$, I Ottaviani $^{1}$, \\ M L Terranova ${ }^{2}$ and F Toschi ${ }^{2}$ \\ ${ }^{1}$ Dipartimento di Fisica and MINAS Laboratory, Università di Roma 'Tor Vergata', I-00133Roma, Italy \\ 2 Dipartimento di Scienze e Tecnologie Chimiche and MINAS Laboratory, Università di Roma 'Tor \\ Vergata', I- 00133 Roma, Italy
}

Received 9 September 2011, in final form 6 February 2012

Published 24 February 2012

Online at stacks.iop.org/JPhysD/45/105306

\begin{abstract}
We report on experiments conducted on single-walled carbon nanotube bundles aligned in chains and connected through a natural contact barrier. The dependence upon the temperature of the transport properties is investigated for samples having different characteristics. Starting from two bundles separated by one barrier deposited over four-contact probes, we extend the study of the transport properties to samples formed by chains of several bundles. The systematic analysis of the properties of these aggregates shows the existence of two conduction regimes in the barrier. We show that an electrical circuit taking into account serial and parallel combinations of voltages generated at the junctions between bundles can model the samples consistently.
\end{abstract}

(Some figures may appear in colour only in the online journal)

The interest for carbon nanotubes (CNTs) in the electronics industry has grown considerably since the fabrication of transistors and diodes based on metal or semiconducting CNTs [1]. Although devices based on a single CNT have been proposed and analysed in several contexts [2], it is predictable that aggregates [3] of CNTs can also offer interesting counterparts for applications. The properties of the aggregates are more complex to handle and to interpret with respect to the isolated nanotubes; however, their properties are surely very interesting and appealing both for fundamental and applied physics purposes [4]. Of particular interest is the role played by the junctions between the bundles interfaces which act as barriers for the charge carriers. This has been observed in the case of CNT tangles under external pressure [5] or when comparing the transport properties of different aggregates obtained using different growth processes [6]. All these experiments show that the modification of the barriers at the interfaces strongly affects the electrical properties of the aggregate of bundles with the result of modifying the conductivity behaviour of the whole material.

It is the purpose of this paper to show that single-walled carbon nanotube (SWCNT) bundles systems have properties which can be well interpreted in terms of physics of transport phenomena. We report on measurements performed on bundles (containing semiconducting and metallic SWCNTs) aligned along the direction of an external bias current. The temperature dependence of the transport properties of the samples exhibits an ohmic-non-ohmic transition in the current-voltage $(I-V)$ characteristics at temperatures below $80 \mathrm{~K}$. Evidence is shown that the properties of the aggregates can be interpreted in terms of elementary junctions between bundles.

Bundles of semiconducting and metallic SWCNT produced by Cheap Tubes Inc.(www.cheaptubesinc.com) (purity $>90 \mathrm{wt} \%$, ash $<1.5 \mathrm{wt} \%$ ) were deposited on insulating $\mathrm{SiO}_{2}$ substrates where metallic $\mathrm{Au}$ contacts had been previously patterned with four lead configurations as shown in the inset of figure $1(a)$. We call this contact configuration NTPR1 (Nano Tube PRobe 1) in order to distinguish it from another configuration that will be described later; in all cases, the transport measurements herein presented are performed by a four-probe technique. This technique allows us to exclude any effect due to the electrical contact resistance limiting our measured $R$ values to that of the bundles and the junctions at the interfaces. Among them, the junction plays a prominent role because of the presence, inside the bundles, of metallic SWCNT which shorts the current path all along the bundles whereas the junctions act as an insulating barrier. Moreover, it is reasonably to assume that the junctions inside each bundle, eventually formed at the interfaces between different CNTs, 


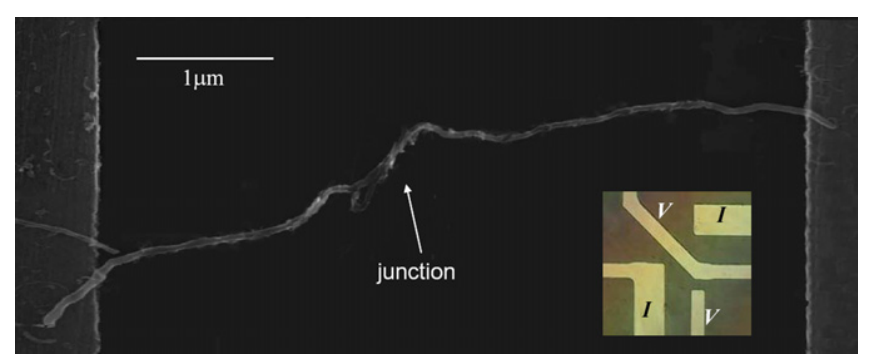

(a)

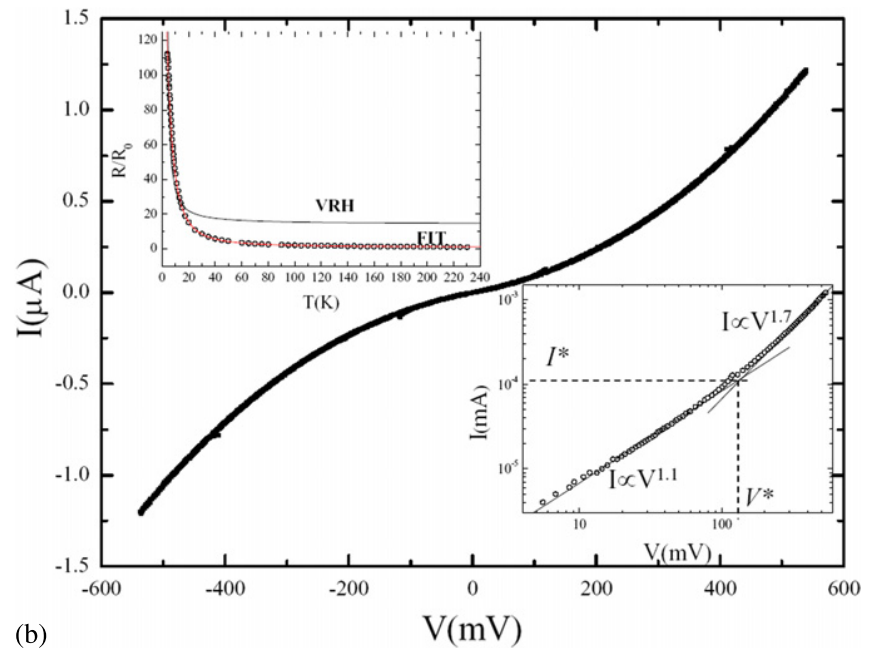

Figure 1. (a) SEM image showing a two bundles chain for NTPR1 contact pattern with the arrow indicating the position of the junction; inset: schematic of the four leads configuration. (b) the current-voltage characteristics measured for the sample shown in (a) at $T=4 \mathrm{~K}$; upper inset: normalized $R-T$ measurements for the sample shown in $(a)$ with respect to the resistance value at $T=240 \mathrm{~K}$, the lower and upper lines are fits to the data following the FIT and the VRH model, respectively; lower inset: same data shown in the main panel but in double logarithmic scale. The fits are obtained by power law with different exponents in the different regimes. The method used to determine $I^{*}$ and $V^{*}$ is also indicated.

have a negligible effect on the charge carriers' motion with respect to the junctions at the interface between different bundles [7]. Taking into account all these considerations, one can assume that the measured voltage drop is mainly confined across each bundle-bundle junction.

The voltage probes distance is fixed at $l_{V}=5 \mu \mathrm{m}$ and the bundles are aligned along the current direction by a dielectrophoretic technique described elsewhere [4]. The length of each bundle ranges between 2 and $3 \mu \mathrm{m}$ and their diameter is typically of the order of $D=100 \mathrm{~nm}$; thus, for this probing configuration even a chain formed by few bundles (in principle even two) can connect the voltage electrodes. The scanning electron microscopy (SEM) analysis confirms the expected length and diameter of the bundles and the formation of contact junctions. In figure 1( $a$ ) we show an example of junction between two bundles: here an arrow indicates the contact area between the bundles. This junction is supposed to be formed by the action of van der Waals forces between the graphenic surfaces with a consequent potential barrier formation [7].

Transport measurements have been performed in a high vacuum cryocooler whose nominal cooling power at the cold finger, to which the samples are secured by metallic silver paste, is $0.5 \mathrm{~W}$ at $T=4.2 \mathrm{~K}$. A normalized resistance (with respect to its value $R_{0}$ at $T=240 \mathrm{~K}$ ) versus temperature $\left(R / R_{0}-T\right)$ dependence of the sample of figure $1(a)$ in the NTPR 1 contact configuration, measured with a bias current of $10 \mathrm{nA}$, is reported in the upper inset of figure $1(b)$. We see that at temperatures below $30 \mathrm{~K}$ the resistance of the sample has a sharp rise like ordinary semiconductors [8] evidencing a strong semiconducting structural component in the sample. The best fit line is obtained by the fluctuation induced tunnel (FIT) [9] model, based on a tunnel mechanism of the charge carriers across a potential barrier enhanced by thermal fluctuations, and is labelled FIT in the inset. In the case of CNTs, this barrier is assumed to be formed at the connection between the bundles [7], shown by the arrow in figure 1(a) for our sample. The FIT model predicts a temperature dependence of the electrical resistance given by $R=R_{0} \mathrm{e}^{T_{1} /\left(T+T_{2}\right)}$ where $T_{1}$ is proportional to the potential barrier, $T_{2}$ is the temperature below which FIT regime is active and $R_{0}$ is the resistance at a given temperature. Setting $R_{0}=12.7 \mathrm{k} \Omega$ (the resistance of the sample at $240 \mathrm{~K}$ ) in our case $T_{1}=204 \mathrm{~K}$ and $T_{2}=30 \mathrm{~K}$ give the best fit of the $R-T$ data in agreement with previous results [10]. It is worth noting that the variable range hopping (VRH) model [8], successfully employed in several CNTs systems, does not fit our experimental data in the whole temperature range investigated. A result of a fitting attempt by the VRH model is also shown in the upper inset of figure $1(b)$ by the curve labelled VRH. This curve represents the best fit of our data following the VRH model, according to which $R=R_{0} \mathrm{e}^{\left(T_{0} / T\right)^{\gamma}}$, obtained with $\gamma=1 / 2$ and $T_{0}=17.35 \mathrm{~K}$. Changing the value of $\gamma$ (setting it equal to $1 / 2,1 / 3$ or $1 / 4$, as allowed by the VRH model) does not improve significantly the quality of the fit.

The $I-V$ characteristic measured at $T=5 \mathrm{~K}$ is reported in the main panel of figure $1(b)$. The curve is rather symmetrical showing that neither metallic contacts nor (insulating) substrate influence the Fermi energy level position with respect to the conduction and valence bands inside the bundles [11]; the nonlinearity in this $I-V$ characteristic was not present at room temperature and we could record it only below $80 \mathrm{~K}$. Using the data in the upper inset of figure $1(b)$, given the value of the resistance $R_{0}=12.7 \mathrm{k} \Omega$ measured at $T=240 \mathrm{~K}$ in the low bias $(I=10 \mathrm{nA})$ regime, from the bundles dimensions measured by SEM, we estimate a resistivity of our sample formed by two connected bundles of $2.3 \mathrm{k} \Omega \mu \mathrm{m}$ at $T=4 \mathrm{~K}$. It is worth noting that the nonlinearity observed in the $I-V$ characteristic appears around $100 \mathrm{mV}$ which rules out effects generated by inter-CNTs contacts inside the bundles: these junctions indeed do generate gaps, but at voltages which are two orders of magnitude lower [1,2].

Plotting the data of the main panel of figure $1(b)$ in double logarithmic scale we obtain the plot reported in the lower inset of figure $1(b)$; from displaying the data in such scales more insight can be gained. The $I-V$ characteristic shows that two straight lines with different slopes can fit the data in the low and high bias current region. The crossing of the two straight lines at the voltage of $V^{*}=140 \mathrm{mV}$ and current $I^{*}=0.1 \mu \mathrm{A}$ provides an indication on the separation between 
the two different transport regimes. Both the slopes are fitted with a power function $I \propto V^{\alpha}$ with the exponent $\alpha$ being 1.1 and 1.7, respectively, for the low and high bias current part of the characteristic. We observed the same features shown in the lower inset of figure 1(b) on dozens of samples and observed that increasing the distance between the voltage electrodes, the $I-V$ characteristic in the lower inset of figure $1(b)$ would scale up in voltage and the values of $V^{*}$ resulted to be increasing function of the distance between the electrodes. For this reason we decided to change the probe design in order to investigate systematically the properties of the aggregates upon the distance between the voltage probing electrodes.

We stepped then to the contacts pattern NTPR 2 shown in the inset of figure $2(a)$ : here we positioned 'inner' voltage electrodes $(100 \mu \mathrm{m}$ long) between the current electrodes (leftmost and rightmost in the figure) at positions multiples of $20 \mu \mathrm{m}$ (respectively $20 \mu \mathrm{m}, 40 \mu \mathrm{m}, 60 \mu \mathrm{m}$ and $80 \mu \mathrm{m}$ ). The CNT aggregates are deposited all over the contact pattern and are aligned in the direction orthogonal to the electrodes. Figure 2(a) shows a SEM image of two parallel bundles, about $2 \mu \mathrm{m}$ apart, connected to one contact pad while the area of the square with the white perimeter is a zoom of a portion of the sample between the probes. We assume, without loss of generality, that the nature of the force governing the contact junction between two different bundles inside the aggregates of figure 2(a) is the same as in the case of two isolated bundles shown in figure 1(a), i.e. van der Waals interaction.

It is worth noting that we herein investigate 'macroscopically' the effects of the junctions between bundles and show the consistent scaling properties of the aggregate as a function of the number of junctions themselves. The only assumption in the interpretation of our measurements is that the nature of the forces in a two bundles junction (figure 1(a)) is the same as in the case of the aggregate of bundles (figure 2(a)). High resolution TEM (HRTEM) analysis of the junctions between bundles could perhaps provide specific structural information, however, at the present level we just rely on macroscopic information consistently sided by the SEM analysis.

In figure $2(b)$ we show the temperature dependence of the $I-V$ characteristics of the aggregates recorded probing, in the NTPR 2 configuration, the voltage between electrodes spaced $20 \mu \mathrm{m}$ apart: we can clearly see the effect mentioned above, namely, that the nonlinearity of the $I-V$ curves starts below $80 \mathrm{~K}$. Also, we can see that when decreasing the temperature, the 'asymptotic' resistance, namely the resistance measured for high voltages decreases as well. The lines are the best fit of the data at $T=4 \mathrm{~K}$ using the same expressions of the lower inset of figure $1(b)$ for the single junction. The different slopes in the $I-V$ characteristics indicate a non-ohmic behaviour of the transport mechanism in the CNTs which is more evident at higher bias current where the difference with the expected ohmic exponent $(\alpha=1)$ increases. We note that the 'gap' existing between the characteristics at 25 and $35 \mathrm{~K}$ is due to the fact that, as we can see in the top-left inset of figure $1(b)$, in this temperature region the resistance undergoes relevant variations.

The main panel of figure 2(c) shows the $I-V$ curves of a sample in the NTPR2 electrode configuration at $T=4 \mathrm{~K}$.

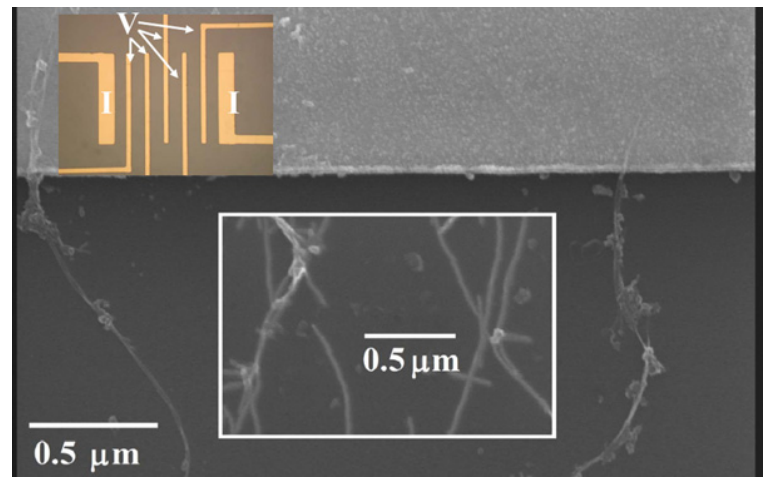

(a)
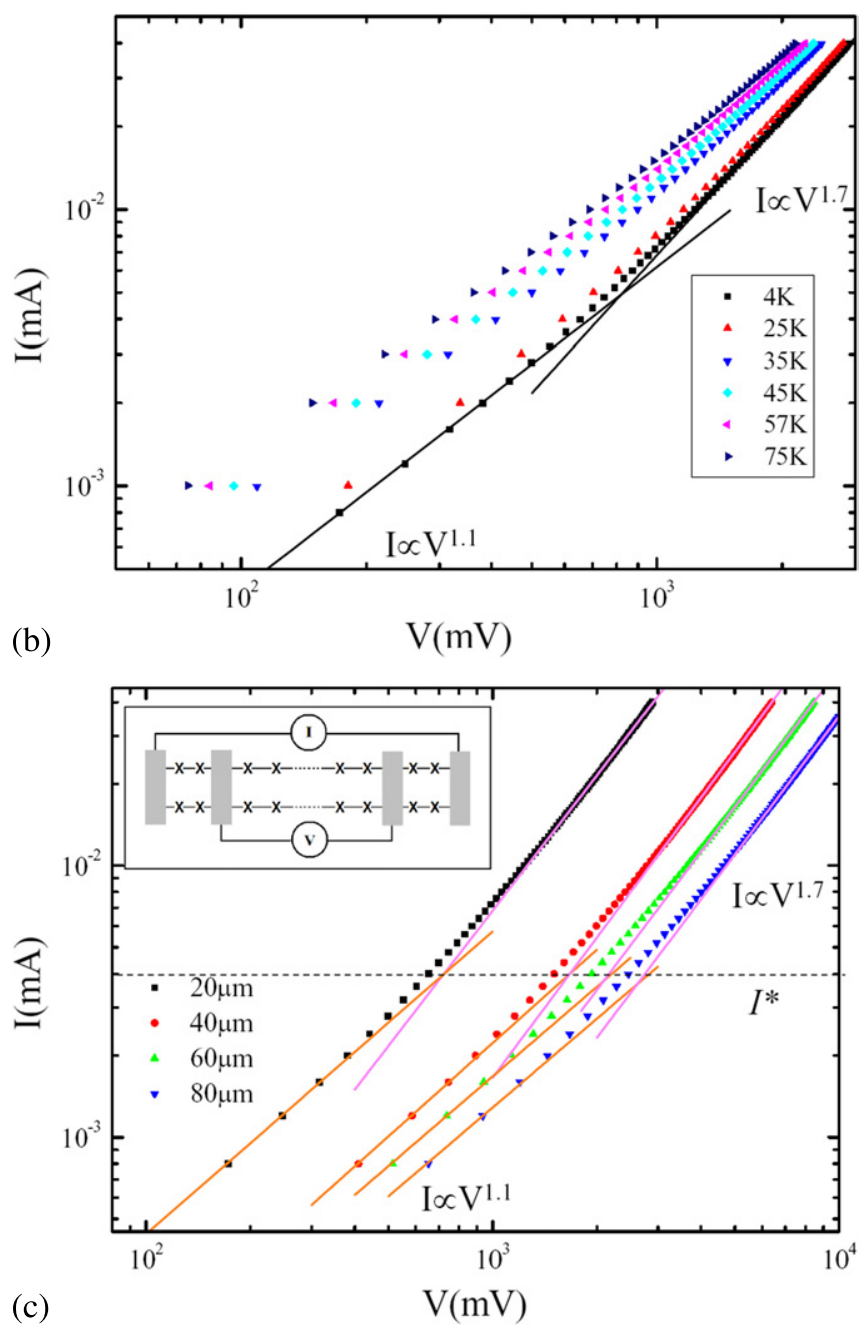

Figure 2. (a) SEM image showing two parallel chains departing from the Au contact pad for NTPR2. The inset shows the bundles in a region between the electrodes. The upper inset shows an optical image of the current external electrodes and the inner voltage electrodes spaced at distances of $20 \mu \mathrm{m}$ each. (b) The $I-V$ characteristics of the aggregate contacted at $L=20 \mu \mathrm{m}$ distance at different temperatures. The lines are fit to the data at $T=4 \mathrm{~K}$ obtained using the same expression used in the lower inset of figure $1(b)$. (c) The $I-V$ characteristics of the aggregate measured using the different voltage electrodes at $T=4 \mathrm{~K}$. The fits to the data are obtained using the same expression of figure $2(b)$; the dashed line indicates that the change in the slope happens at the same current value for the different electrode distances. Inset: sketch of the series/parallel resistance model assumed for the aggregate deposited between two electrodes. 
These characteristics are obtained probing the voltages at the different lengths established by the contact pattern design (20, $40,60$ and $80 \mu \mathrm{m})$. We can see that the change in the slope is similar to that observed for the single junction but it is recorded now for increasing voltage values and scales with the electrodes distance. The current value defined in the lower inset of figure $1(b)$ as the point where the two straight lines fitting the data cross each other is now $I^{*} \cong 4 \mu \mathrm{A}$; we note that this value is the same for all the measured probing distances but it is higher with respect to the case of the single junction shown in figure $1(b)$. The lines in the figure represent the best fit of the data in the two current bias regimes using the same power law function as in figure $1(b)$ : we note that all the fittings provide the same exponents (1.1 in the low bias regime and 1.7 in the high bias region). Note that in this case the 'gap' existing between the characteristics taken at 20 and $40 \mu \mathrm{m}$ is likely due to the fact that between the density of bundles deposited in the fabrication process is not uniform all over the probing electrodes: in other samples we have seen a 'gap' in correspondence to two other different probing lengths (say $60 \mu \mathrm{m}$ and $80 \mu \mathrm{m}$ for example).

The $I-V$ dependences of figure 2(c) can be understood by schematically modelling the bundle aggregate aligned between two consecutive voltage electrodes as a system of $n_{\mathrm{s}}$ series and $n_{\mathrm{p}}$ parallel (nonlinear) circuit elements. In the sketch of the inset of figure 2(c) the crosses indicate the junctions between the bundles. We show first that it is reasonable to assume that the aggregates connecting the electrodes can be viewed as sketched in the inset. We will show it in the limit of low current bias but this peculiar choice of current bias does not limit the generality of the demonstration. The SEM analysis tells us nanotubes are roughly $2-3 \mu \mathrm{m}$ long and therefore we estimate that between two electrodes separated by a distance $L=20 \mu \mathrm{m}$ we could have a series connection of (7-10) bundles and therefore $n_{\mathrm{s}}=8$ is a reasonable guess. Measuring the resistance from the $I-V$ characteristic in the low current limit we obtained for the case $L=20 \mu \mathrm{m}$ the value $R_{20}=223 \mathrm{k} \Omega$. According to our model $R_{20}=\left(n_{\mathrm{s}} R\right) / n_{\mathrm{p}}$ (where $R=1.4 \mathrm{M} \Omega$ is the resistance of a single junction as measured from the $I-V$ curve of figure $1(b)$ at $T=4 \mathrm{~K}$ ) which gives $n_{\mathrm{p}} \cong 50$ as the number of parallel bundles chains. This number is consistent with the SEM analysis, which shows an average distance of $2 \mu \mathrm{m}$ between two nearest bundles connected perpendicularly to the $100 \mu \mathrm{m}$ length electrodes.

We also note that the current value $I^{*} \cong 4 \mu \mathrm{A}$ is about 40 times higher than the $I^{*}$ value measured in the case of the single junction of figure $1(a)(0.1 \mu \mathrm{A})$ : the scaling of the current is of the same order of magnitude of that predictable from the above modelling (from which we expect a factor 50). Increasing the distance between the electrodes, a number of $m \cdot n_{\mathrm{s}}$ (with $m=1, \ldots 4$ ) resistances are added in series whereas the number of parallel $n_{\mathrm{p}}$ chains remains the same allowing the same current $I^{*}$ (indicated by the dashed line in figure $2(c)$ ) and different voltages measured in the different electrodes distance in the NTPR2 configuration.

Measuring the voltage corresponding to the $I^{*}$ value of figure $2(c)$, we obtain the values $V^{*}=1.67 \mathrm{~V}, 2.23 \mathrm{~V}$ and $2.87 \mathrm{~V}$ for $L=40 \mu \mathrm{m}, 60 \mu \mathrm{m}$ and $80 \mu \mathrm{m}$, respectively. All these values are in the ratio $2.2,3.0$ and 3.9 with $V^{*}=740 \mathrm{mV}$ which corresponds to the case $L=20 \mu \mathrm{m}$ confirming the $m \cdot n_{\mathrm{s}}$ (with $m=1, \ldots 4$ ) rule with a good approximation. This analysis confirms that the nonlinearities observed in the $I-V$ characteristics in figures $1(b), 2(b)$ and $2(c)$ are generated by elementary junctions formed at the interfaces between the bundles. It is worth noting that both the properties of the interface between the bundles leading to the structural formation of the barrier and the same contacts between the bundles and the metal contact probes might have a very intriguing physical nature [12]. The discrepancy between the expected and the estimated $m$ values reflects on the presence of the larger gap at $I^{*}$ level present in the case of $L=20 \mu \mathrm{m}$ and $L=40 \mu \mathrm{m} I-V$ curves of figure 2(c) which can be attributed to the difficulty in depositing exactly the same number of bundles between two consecutive contacts. However, our four-probe configuration 'averages' out the property of the interbundle barriers displaying characteristics of the junctions which are robust and reliable at macroscopic level. In what follows we provide two more quantitative evidences in favour of this argument.

In figure 2(c) we show $I-V$ characteristics measured at $4 \mathrm{~K}$ which can be fitted by different values of resistances in the low and high bias current regime. These $I-V$ have been obtained probing the voltages at the different lengths, as indicated in the figure. In order to test the scaling of this behaviour with the number of junctions we calculate the resistance from the slope of the $I-V$ characteristics (differential resistance) for all the voltage electrode distances both in the low and in the high bias current regime. Figure 3 shows the $R$ versus $L$ dependence for all the investigated temperatures in the case of low bias current and high bias current regimes (figures 3(a) and $(b)$, respectively). We can see that in both the cases a linear regression is a good approximation for the $R$ versus $L$ dependence which confirms that the voltage drops that we are recording across the aggregate are generated by the sum of the potential differences generated at the individual junctions between bundles.

As a further check that the physical properties of the arrays can be interpreted by the same mechanism explaining the behaviour of 'single' junctions between bundles, we have extracted from the data of figure 3(a) the differential resistance, in the limit of low bias current, as a function of the temperature for all the values of the electrode distance $L$. The results are shown in figure 4 where the lines are fit to the data based on the FIT model. As shown in the inset of figure 1(b), the FIT theory gives the framework of the transport mechanism in the case of the single junction and reasonably good agreement with the experimental data also in the case of the arrays of junctions is a further confirmation of the correctness of our model.

In order to provide a complete analysis of the transport properties of our samples we shall now estimate the selfheating of the samples due to the bias current (Joule heating). We perform the calculation in the case of the single junction in figure 1(a) using the expression [13] $T(x)=T_{0}+$ $\left(p^{\prime} / g\right)\left[1-\cosh \left(x / L_{H}\right) / \cosh \left(L / 2 L_{H}\right)\right]$ where $T_{0}=4 \mathrm{~K}$ is the temperature at the contacts, $x$ is the coordinate along the tube, $L\left(=l_{V}=5 \mu \mathrm{m}\right)$ its length, $p^{\prime}=I \cdot V / L$ is the 


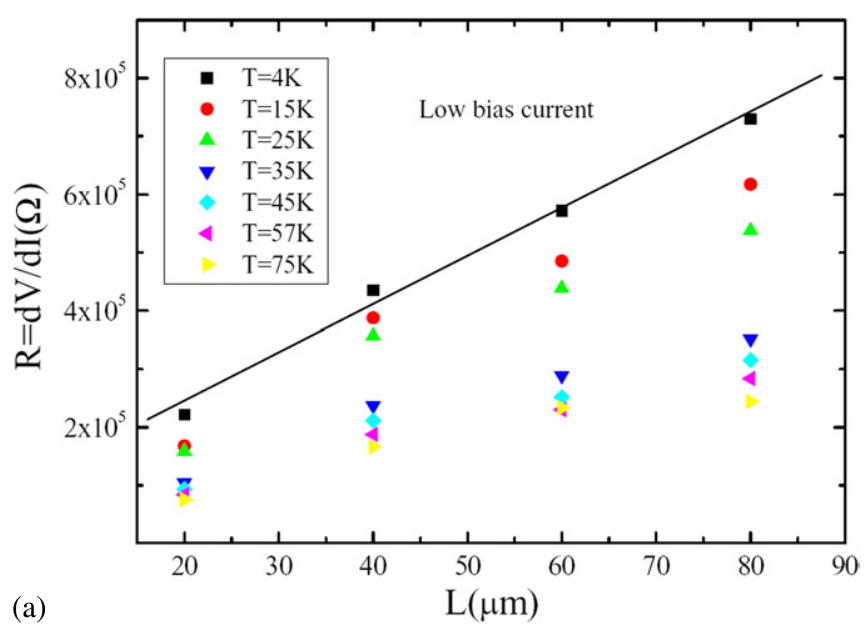

(a)

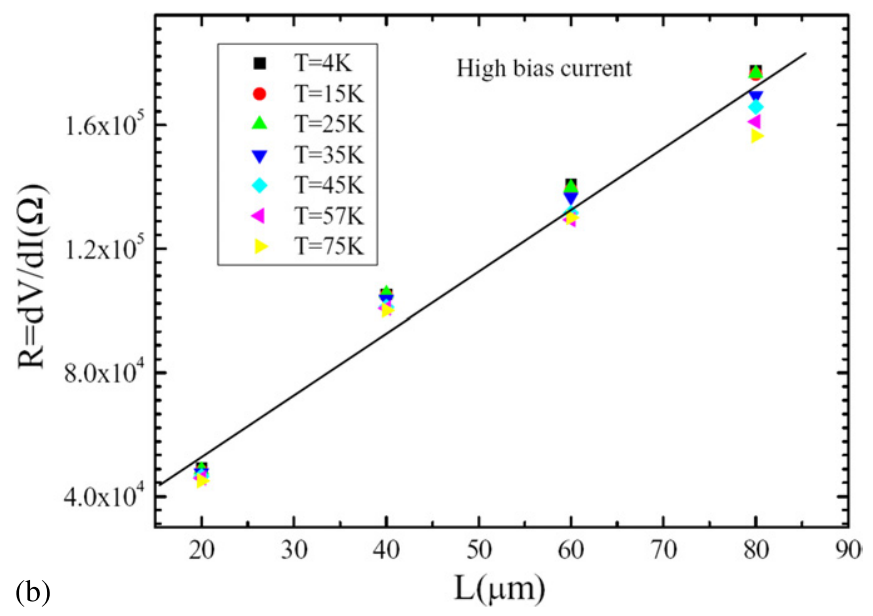

Figure 3. (a) Differential resistance versus $L$ measured by the $I-V$ curves acquired for all the electrode configuration at different temperatures and in the regime of low bias current. (b) same as (a) but in the high bias current regime. In both the figures the straight lines are guide to the eyes for the $T=4 \mathrm{~K}$ data.

electric power per unit length in the tube due to the bias current, $g$ is the thermal conductance per unit length between the bundles and the substrate and $L_{H}=(k A / g)^{1 / 2}$, with $k$ the thermal conductivity and $A$ the cross-sectional area of the sample, is the characteristic thermal length of the bundles. We estimated $p^{\prime}=0.12 \mathrm{~W} \mathrm{~m}^{-1}$ by the maximum current and voltage values used for this sample in the $I-V$ measurement reported in the lower inset of figure $1(b) I=1 \mu \mathrm{A}$ and $V=600 \mathrm{mV}$. The estimate of the other parameters inside the expression for $T(x)$ has been done using literature data and taking into account their temperature dependence [14]. The calculated value of $g=0.65 \mathrm{~W} \mathrm{~m}^{-1} \mathrm{~K}^{-1}$ is obtained by equation (5) in the [13] taking into account that, apart from some geometrical factors specified in the reference, it depends on the thermal conductivity of the $\mathrm{SiO}_{2}$ substrate that at $T=4 \mathrm{~K}$ is approximatively $5 \times 10^{-2} \mathrm{~W} \mathrm{~m}^{-1} \mathrm{~K}^{-1}$ [15]. Finally, the value $L_{\mathrm{H}}=2.7 \times 10^{-8} \mathrm{~m}$ is obtained by the value of the thermal conductivity of aligned SWCNT films that at $T=4 \mathrm{~K}$ is about $k=20 \mathrm{~W} \mathrm{~m}^{-1} \mathrm{~K}^{-1}$ [16]. Substitution of these values in the expression of $T(x)$ give a maximum change in the temperature between the Au leads and the centre of the sample of $\Delta T \approx 0.2 \mathrm{~K}$ which is less than our experimental

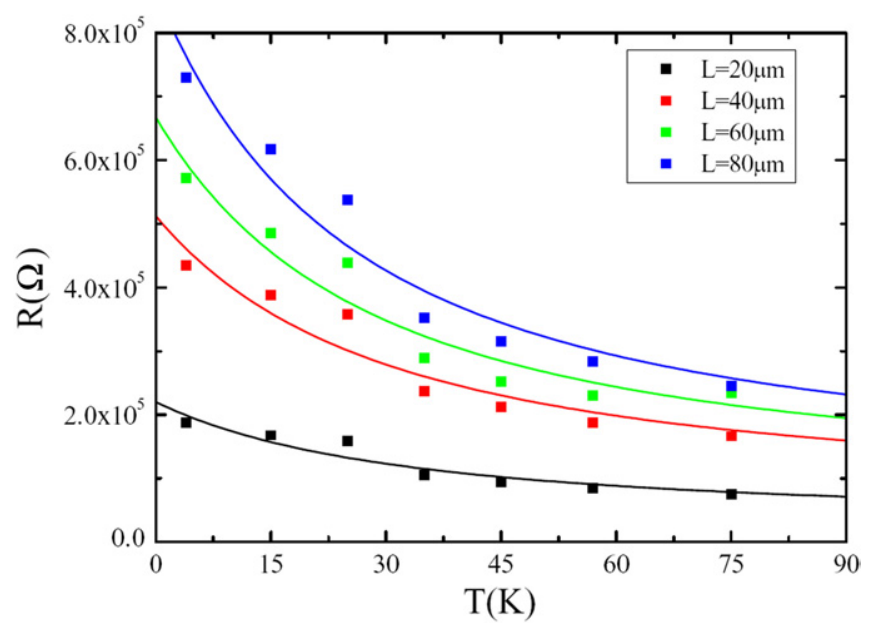

Figure 4. Differential resistance as a function of the temperature obtained by the $I-V$ characteristics in the limit of low bias current for all the values of $L$. The lines are fit to the data following the FIT model. The fitting parameters obtained from the fit of the normalized curves with respect to the $R$ value at $T=240 \mathrm{~K}$ are: $T_{1}=170 \mathrm{~K}, 165 \mathrm{~K}, 162 \mathrm{~K}, 157 \mathrm{~K}$ and $T_{2}=80 \mathrm{~K}, 76 \mathrm{~K}, 73 \mathrm{~K}, 68 \mathrm{~K}$ for $L=20 \mu \mathrm{m}, 40 \mu \mathrm{m}, 60 \mu \mathrm{m}$ and $80 \mu \mathrm{m}$, respectively.

uncertainty. Because the same heating can be considered in the case of the NTPR2 configuration (formed by $n_{\mathrm{p}}$ parallel bundles of the same kind) we assume that the cooling power of $0.5 \mathrm{~W}$ of our cooling system is sufficient to exclude any Joule heating effect as the cause of the observed change in the slope of the $I-V$ characteristics.

In our samples there exist resistances between the CNTs and silver paste, between the CNTs and gold layer, between CNT and the CNT inside a bundle and between two different CNT bundles. While the four-contact probe allows us to exclude the influence of contact resistances in the measurements, it surely does not enable us to exclude the possibility that the observed phenomena could be due to some kind of inter-CNT tunnelling inside the bundles. One possibility to check that would be to four-contact probe a single bundle and measure its $I-V$ curve. Unfortunately, this is not a simple experimental task since the diameter of a bundle is of the order of $100 \mathrm{~nm}$ and the length of a bundle is only a couple of micrometres. Thus, in order to obtain a further proof that the phenomena described herein depend upon the junctions between bundles and are not generated inside a single bundle we show in figure 5 an $I-V$ curve of a $100 \mu \mathrm{m}$ diameter CNT fibre whose characteristics are detailed in a previous publication [6]. Inside these fibres the SWCNTs are isolated and not organized in bundles and therefore each fibre can be viewed as a single isolated thick bundle. We see that in the case of the fibres the $I-V$ shows an ohmic behaviour in the temperature and voltage regimes investigated for the bundles aggregates meaning that a single bundle, even a rather big one, does not display changes in the slope of its $I-V$ curve.

In conclusion, we have reported on the macroscopic investigation of tunnelling barrier effects in aggregates of SWCNT bundles. We have provided experimental evidence that the transport properties of the aggregates do not follow 


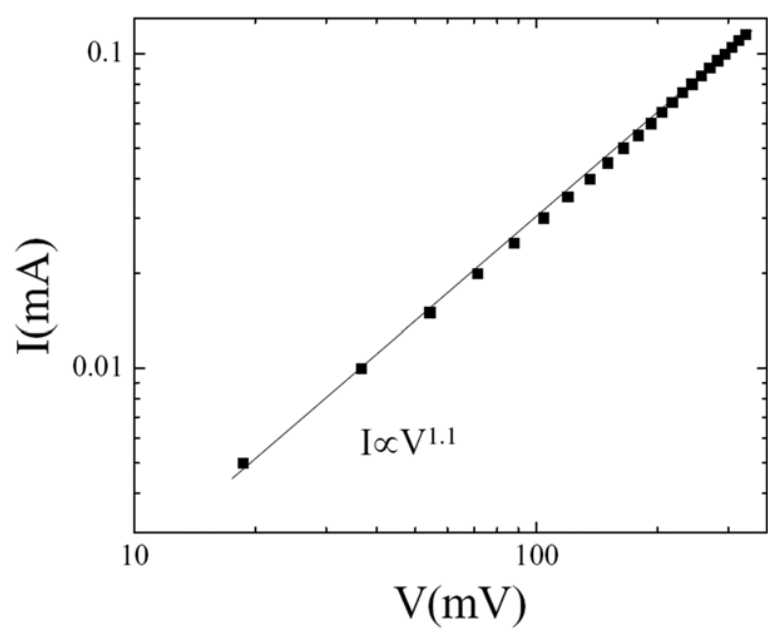

Figure 5. The $I-V$ characteristic of a $100 \mu \mathrm{m}$ - thick CNT fibre taken at $5 \mathrm{~K}$. We see that the $I-V$ does not display nonlinearities. Since a CNT fibre can be viewed as a single big bundle the figure shows that a single bundle does not display a change in slope in the $I-V$ curve and that the nonlinearities can safely be attributed to junctions between bundles. The plot of this figure was extracted from data presented in [6].

ohmic behaviour; however, we have also shown how the properties of the aggregates scale with the length of the aggregates, namely with the number of junctions between the bundles contacting the electrodes. The $I-V$ characteristics of the samples can be well understood in terms of a simple electrical model accounting for series-parallel connections of the junctions present in the nanotube bundles chains contacting the probe electrodes. Our results show that even a complex system such as a nanotube bundles aggregate might have solid properties to be considered for potential applications; it is natural to candidate the investigated aggregates as elements with reproducible electrical characteristics for interconnect applications [17]; however, the evidence of a temperatureinduced nonlinearity in the $I-V$ singularities is also a stimulating result in terms of devices physics and sensors. This nonlinearity is robust enough below $77 \mathrm{~K}$ (the liquid nitrogen temperature) which would imply cooling the samples in liquid nitrogen; this is a low cost cryogenic requirement which is increasingly considered today for operation of electronic devices.

\section{Acknowledgment}

The financial support of the GESTO program of the Regione Lazio (Italy) is gratefully acknowledged.

\section{References}

[1] Tans S J, Verschueren A R M and Dekker C 1998 Nature 39349

Rinkiö M, Johansson A, Paraoanu G S and Törma P 2009 Nano Lett. 9643

[2] Bachtold A, Hadley P, Nakanishi T and Dekker C 2001 Science 2941317

Weitz R T, Zschieschang U, Forment-Aliaga A, Kalblein D, Burghard M, Kern K and Klauk H 2009 Nano Lett. 91335

[3] Saito R, Dresselhaus G and Dresselhaus M S 1998 Physical Properties of Carbon Nanotubes (London: Imperial College Press)

[4] Terranova M L, Lucci M, Orlanducci S, Tamburri E, Sessa V, Reale A and Di Carlo A 2007 J. Phys.: Condens. Matter 19225004

[5] Allaoui et al 2009 Scr. Mater. 61628

[6] Salvato M, Lucci M, Ottaviani I, Cirillo M, Orlanducci S, Tamburri E, Guglielmotti V, Toschi F, Terranova M L and Pasquali M 2011 J. Phys.: Condens. Matter 23475302

[7] Furer M S et al 2000 Science 288494

[8] Mott N F and Davis E A 1979 Electronic Processes in Non-Crystalline Materials (Oxford: Clarendon) (see in particular chapter 6)

[9] Sheng P, Sichel E K and Gittleman J I 1978 Phys. Rev. Lett. 4001197

[10] Salvato M, Cirillo M, Lucci M, Orlanducci S, Ottaviani I, Terranova M L and Toschi F 2008 Phys. Rev. Lett. 101246804

[11] Kim J, Lee J, Oh H, Yoo K H and Kim J J 2001 Phys. Rev. B 64161404

[12] San-Huang Ke et al 2007 Phys. Rev. Lett. 99146802

Saha K K et al 2010 Phys. Rev. Lett. 105236803

[13] Pop E, Mann D A, Goodson K E and Dai H 2007 J. Appl. Phys. 10193710

[14] Santavicca D F, Chudow J D, Prober D E, Purewal M S and Kim P 2010 Nanoletters 104538

[15] Stephens R B 1973 Phys. Rev. B 82896

[16] Hone J, Llaguno M C, Nemes N M, Johnson A T, Fisher J E, Walters D A, Casavant M J, Schmidt J and Smalley R 2000 Appl. Phys. Lett. 77666

[17] Yun-Hi Lee et al 2001 Adv. Mater. 131371

Robertson J, Zhong G, Telg H, Thomesen C, Warner J H, Briggs G A D, Dettlaff-Weglikowska U and Roth S 2008 Appl. Phys. Lett. 93163111 